\title{
Influence of Beta-2-Adrenergic Receptor GIn27Glu Polymorphism on the Autonomic Heart Rate Modulation in Young Sedentary and Physically Active Women
}

\author{
Rebelo Ana Cristina Silva ${ }^{1}$, Melo Aryanne Batista Soares ${ }^{2}$, Salviati Mariana Rodrigues 3 , \\ Verlengia Rozangela ${ }^{3}$, Vale Arthur Ferreira Do ${ }^{1}$, Nogueira Yanley Lucio ${ }^{4}$, \\ Tamburús Nayara Yamada ${ }^{5}$, Kunz Vandeni Clarice ${ }^{6}$, Silva Ester Da ${ }^{5}$ \\ ${ }^{1}$ Department of Morphology, Biological Sciences Institute, Federal University of Goiás, Goiânia, Brazil \\ ${ }^{2}$ Center for Neuroscience and Cardiovascular Physiology Research, Department of Physiological Sciences, Biological Sciences Institute, \\ Federal University of Goiás, Goiânia, Brazil \\ ${ }^{3}$ Humana Performance Research Group - College of Health Sciences (FACIS), Methodist University of Piracicaba (UNIMEP), Piracicaba, \\ Brazil \\ ${ }^{4}$ Faculty of Medicine, Federal University of Goiás, Goiânia, Brazil \\ ${ }^{5}$ Department of Physiotherapy, Federal University of Sao Carlos, Sao Carlos, Brazil \\ ${ }^{6}$ Adventist University of Sao Paulo, Engenheiro Coelho, Sao Paulo, Brazil
}

\section{Email address:}

anacristina.silvarebelo@gmail.com (R. A. C. Silva)

\section{To cite this article:}

Rebelo Ana Cristina Silva, Melo Aryanne Batista Soares, Salviati Mariana Rodrigues, Verlengia Rozangela, Vale Arthur Ferreira Do, Nogueira Yanley Lucio, Tamburús Nayara Yamada, Kunz Vandeni Clarice, Silva Ester Da. Influence of Beta-2-Adrenergic Receptor Gln27Glu Polymorphism on the Autonomic Heart Rate Modulation in Young Sedentary and Physically Active Women. American Journal of BioScience. Vol. 3, No. 5, 2015, pp. 190-196. doi: 10.11648/j.ajbio.20150305.15

\begin{abstract}
Background: Studies have shown an interaction between the autonomic heart rate (HR) modulation and genetic polymorphisms. Nevertheless, it is yet unclear how a particular genetic polymorphism may determine a pattern of autonomic HR control, especially regarding the role of physical exercise. The objective was to investigate the influence of of Beta-2Adrenergic ( $\beta 2-\mathrm{AR})$ Gln27Glu polymorphisms and exercise on HRV of young Brazilian women. Methods: The group was selected as Sedentary Group (SG; $n=88$ ) and physically Active Group (AG; $n=99)$. The $\beta 2$-AR polymorphisms were analyzed using PCR-RFLP. The amplicons were subjected to electrophoresis on a $10 \%$ acrylamide gel and stained with ethidium bromide. The HR was registered in real time for 15 minutes, in the supine-position. The heart rate variability (HRV) was analysed using Shannon's entropy (SE), conditional entropy (complexity index [CInd] and normalized CInd [NCI]), and symbolic analysis (0V\%, 1V\%, 2LV\%, and 2ULV\%). Statistical analysis: Analysis of variance (ANOVA) with Fisher's Least. Results: AG presented higher values for SE and $2 \mathrm{ULV} \%$, and lower values for $0 \mathrm{~V} \%$ when compared to $\mathrm{SG}$ ( $\mathrm{p}<0.05$ ). There was no significant difference of any index among genotypes both from the complexity and symbolic analysis. The interaction between genotype and exercise did not significantly affect HRV. Conclusion: These results do not support the concept that the $\beta 2-A R$ Gln27Glu polymorphisms affects the HRV indexes in physically active or sedentary women.
\end{abstract}

Keywords: $\beta_{2}$-Adrenergic Polymorphism, Heart Rate Variability, Non-Linear Analysis, Women, Exercise

\section{Introduction}

Heart activity is largely modulated by the autonomic nervous system, which promotes rapid adjustments in the cardiovascular system during different stimuli (i.e., physical exercise, mental stress and postural change) $[1,2]$. Heart rate variability (HRV) is a non-invasive measure used to analyze the influence of the autonomic nervous parasympathetic contributions to consecutive HR oscillations. Reduced HRV indices are associated with an increased risk of future cardiovascular disease onset [3-5].

Studies have shown a significant genetic influence on a 
variety of HRV measures [6,7]. $\beta_{2}$ adrenergic receptor ( $\left.\beta 2-\mathrm{AR}\right)$ have a pivotal role in the functions of the cardiac autonomic nervous system. Activation of $\beta 1-A R$ provides strong stimulus to increase the frequency and contractility of the heart, whereas the activation of $\beta_{2}$-AR results in smooth muscle relaxation and increased cardiac output with less extent when compared to $\beta_{1}$-AR $[7,8]$.

Considering that the participation and function of $\beta 2-A R$ have been widely recognized, a large amount of research is being done regarding the physiological and physiopathological relevance of polymorphisms of receptors in cardiac insufficiency [9], sistemic arterial hypertension [10], obesity [11-13] and metabolic syndromes [14].

Studies have also observed an interaction between the autonomic heart rate (HR) modulation and genetic polymorphisms. Matsunaga et al. [15] observed a reduction in high frequency (HF) indexes in Japonese men carriers of Glu/Glu genotype. Atala et al. [16] observed by linear analyses (time domain and spectral) in a healthy young population that the $\beta 2-A R$ Gly16Arg polymorphisms can influence intermediate phenotypes, such as HR, cardiac parasympathetic modulation, and baroreflex sensitivity.

It is yet unclear how a particular genetic polymorphism may determine a similar pattern of autonomic HR control from one subject to another, especially regarding the role of physical exercise. It is know that an interaction of several variables such as environmental, morphophysiological, trainability and genetic factors contribute to modifications in the cardiac autonomic modulation [17]. Macho-Azcarate et al. [18] suggest that both lipolysis and fat oxidation promoted by an acute submaximal exercise intervention could be blunted in the $\beta_{2}$-AR polymorphisms of female obese population. However, the association between $\beta_{2}$-AR Gln27Glu and cardiac autonomic modulation in physically active and sedentary young women was not described.

The following study will do a wide evaluation of the autonomic variation of HR based on non-linear analysis. The use of polymorphism analysis will undeniably contribute to create a profile of the population's genotype and understanding of which specific phenotypes are altered and can influence autonomic adaptations during physical activity.

The present study is important because intermediate physiological traits in healthy individuals may be predictive of future disease or a distant phenotype. The phenotype can be modulated by physical exercise, creating a cardioprotective effect, improving autonomic cardiac modulation [19]. Cardiovascular modulation is interconnected with the nonlinear theory which is sensitive in detecting changes in HRV patterns caused by different stimuli, adaptations and/or pathologies [20].

The hypothesis of the present study is that polymorphisms in ADR- $\beta_{2}$ are associated with the autonomic cardiac response; more specifically, physically active women, carriers of the genotype ADR- $\beta_{2}$ Gln $27 \mathrm{Gln}$, may have better HRV indexes when compared with non-carriers. The objective was to investigate the influence of $\beta_{2}$-AR Gln27Glu polymorphisms on HRV of young sedentary and active Brazilian women.

\section{Material and Methods}

The Ethics Committee of the Methodist University of Piracicaba, SP, Brazil (protocol \#43/06), approved the study. All participants gave written informed consent. A group of 187 healthy Caucasian women between 18 to 30 years of age in the Brazilian cohort were selected as Sedentary Group (SG; $\mathrm{n}=88$ ) and physically Active Group (AG; $\mathrm{n}=99$ ). The AG performed continuous physical activity at least 3 days per week (for at least 6 months) at an intensity of $65-90 \%$ of maximum HR with durations of 30-60 minutes in accordance to the American College of Sports Medicine. The inclusion criteria for all subjects were body mass index (BMI) between 19.0 and $24.9 \mathrm{~kg} / \mathrm{m} 2$; ovulation confirmed by serum progesterone above $4.0 \mathrm{mg} / \mathrm{mL}$ on the $21 \mathrm{st}$ day of the menstrual cycle.

An evaluation form containing previous family history of existing pathologies, use of oral contraceptives and physical activity level was filled out on a daily basis. Resting HR was measured with a 12-lead electrocardiogram (ECG). HR and BP were evaluated after 5 min of rest in the supine and sitting positions during two visits to the laboratory during the follicular phase of the menstrual cycle. All subjects were in good health, and their biochemical parameters were within normal range. Subjects showing clinical evidences and/or biochemical signs of hyperandrogenism, cardiac or respiratory disease, hypertension ( $\mathrm{BP} \geq 140 / 90 \mathrm{mmHg}$ ), diabetes mellitus, thromboembolic disease, thyroid diseases, stroke, depression or a history of smoking or alcohol use were excluded from the study. None of the subjects were taking sedatives, antihypertensives, antiarrhythmic or other medication that could affect autonomic control of HR.

Resting HR was measured with a 12-lead ECG and a maximum exercise test was conducted using cardiac auscultation. HR and BP were measured after 5 min of rest in the supine and sitting positions by the Korotkoff auscultatory method every 2 min with a mercury column sphygmomanometer (WanMed São Paulo, SP, Brazil) and a stethoscope (Littman, St. Paul, MN, USA) during two visits to the laboratory.

The experiments were carried out during the morning to avoid differences in response due to circadian changes. Room temperature was maintained at $22^{\circ} \mathrm{C}$ with relative air humidity $40 \%$ to $60 \%$. Subjects were informed of the experimental protocol and instructed to abstain from use of stimulants (coffee or tea) and alcoholic beverages during the 24 hours preceding the test as well as to have a light meal at least 2 hours prior to the measurement. ECG and HR data were obtained from a one-channel heart monitor (Miniscope II, Instramed, Porto Alegre, RS, Brazil) and processed using a Lab-PC + analog-to-digital converter (National Instruments Corp., Austin, TX, USA), which interfaced between the heart monitor and a computer. Signals were recorded in real time after analog-to-digital conversion at a sampling rate of $500 \mathrm{~Hz}$, and the R-R intervals (RRi) (ms) were calculated on a beat-tobeat basis using specific software. To evaluate the effect of body position on HR response and its variability, RRi were recorded at rest over an 8-min period with the subjects in both 
the supine and sitting positions while breathing spontaneously. HRV were assessed in both time and frequency domains. The region of greatest stability for gathering RRi was used for this measurement so that at least 256 consecutive beats were observed [21].

Shannon's entropy (SE) was calculated to qualify the complexity of the pattern distribution. Shannon's entropy is higher when distribution is uniform and lower if a subset of probable standard tests is missing or infrequent (for example, in a Gaussian distribution) [22,23].

Symbolic analysis entails (i) the transformation of a heart period variability series into a sequence of integers (i.e., symbols), (ii) the construction of patterns, (iii) reduction in the number of patterns by grouping them into a small number of families, and (iv) the evaluation of the rates of occurrence of these families. The pattern families were as follows: (i) patterns with no variation [0V], (ii) patterns with one variation [1V], (iii) patterns with two like variations [2LV], and (iv) patterns with two unlike variations [2ULV]. The percentage of occurrence of each pattern was calculated $(0 \mathrm{~V} \%$, $1 \mathrm{~V} \%, 2 \mathrm{LV} \%$ and $2 \mathrm{ULV} \%$ ). Studies with pharmacological blockade and autonomic tests (like the tilt test) have indicated that $0 \mathrm{~V} \%$ and $2 \mathrm{ULV} \%$ represent sympathetic and parasympathetic modulations, respectively $[20,22,23]$.

In this approach, the minimum corrected conditional entropy with respect to past values was taken as a CInd and the index was normalized by the SE of the RR series to obtain a NCI. The larger the complexity index and the normalized complexity index, the higher the complexity and the smaller the regularity. However, neither Shannon's entropy nor the corrected conditional entropy provide information about the predominance of sympathetic or parasympathetic modulation [24].

DNA was isolated from white blood cells with the use of a standard salting-out procedure [25]. ADR- $\beta 2$ Gln27Glu (rs1042714) polymorphisms were determined using polymerase chain reaction (PCR) and restriction fragment analysis. PCR assays were carried out in a Biometra $\mathrm{T}$ Gradient (Whatman Biometra, Göttingen, Germany). Amplification conditions were set as follows: a 10-min initial denaturation at $94^{\circ} \mathrm{C}$, followed by 30 cycles at $95^{\circ} \mathrm{C}$ for 1 $\min , 63^{\circ} \mathrm{C}$ for $1 \mathrm{~min}$ and $72^{\circ} \mathrm{C}$ for $1 \mathrm{~min}$; final extension was conducted at $72^{\circ} \mathrm{C}$ for $10 \mathrm{~min}$. The sequence primers used 5'-GAA TGA GGC TTC CAG GCG TC-3'; and 5'-GGC CCA TGA CCA GAT CAG CA-3'. The 353 bp PCR product was digested with 1 unit of Fnu4HI (New England Biolab Incorporated, Beverly, MA, USA) for 2 hours. Genotyping was as follows: $174,97,55$ and $27 \mathrm{bp}$ for the Gln allele; and 229, 97 and $27 \mathrm{bp}$ for the Glu allele. The amplicons were subjected to electrophoresis on a $10 \%$ acrylamide gel and stained with ethidium bromide. Genotyping quality control was performed as described in detail elsewhere [26]. All genotypes were determined by two independent technicians, the results were entered in the database in duplicate, and $10 \%$ of the samples were randomly reanalyzed.

Statistical analysis

The calculation of sample size was based on the data reported for the population by [27]. Considering $p=.017$ and the probability of a woman found in the population with the characteristic (Gln27 or Glu7). The sample size was $\mathrm{N}=68$, error of $10 \%$ and power of 0.80 . The allele frequencies and genotype distribution were estimated by gene counting. Hardy-Weinberg equilibrium was assessed with the $\chi^{2}$ test using the Arlequin v3.11 software, which uses an expectationmaximization algorithm. Linkage disequilibrium (LD) and haplotype frequencies were estimated with the LD coefficient (Lewontin's Lewontin's D') from each pair of SNPs using Haploview 4.2 software. Statistical Package for the Social Sciences v.21 was used to compare frequencies among groups, adjusted residuals and the power of the test. T-test was used to compare demographic, clinical and laboratory characteristics between the SG and AG groups. The relationship between genotype groups and cardiovascular variables was analyzed using analysis of variance (ANOVA) with Fisher's Least significant difference correction for multiple comparisons $(\mathrm{p}<0.05)$.

\section{Results}

\subsection{Clinical Characteristics}

Baseline characteristics including anthropometric variables, HR, systolic blood pressure (SBP) and diastolic blood pressure (DBP) are shown in Table 1. No significant differences in age, weight, height, BMI, SBP, DBP and HR were found between the AG and SC groups. There were no significant intergroup differences in mean fasting glucose, urea and creatinine $(\mathrm{p}>0.05)$. Mean HR was higher in $S G$ group than in $\mathrm{AG}(\mathrm{p}<0.05)$.

Table 1. Demographic, clinical and laboratory characteristic of young women.

\begin{tabular}{|c|c|c|c|c|}
\hline Variables & Total $(n=187)$ & $\operatorname{AG}(n=88)$ & SG $(n=99)$ & p-value \\
\hline Age, years & $22,7 \pm 7,2$ & $26,3 \pm 6,5$ & $26,9 \pm 7,4$ & 0,58 \\
\hline Weight, $\mathrm{kg}$ & $59,9 \pm 8,3$ & $60,0 \pm 8,4$ & $59,8 \pm 8,0$ & 0,70 \\
\hline Height, cm & $163,2 \pm 13,5$ & $163,5 \pm 6,6$ & $162,8 \pm 17,6$ & 0,65 \\
\hline BMI, kg/m2 & $22,6 \pm 3,6$ & $22,4 \pm 2,5$ & $22,7 \pm 4,4$ & 0,69 \\
\hline HR supine, bpm & $67,2 \pm 10,3$ & $58,9 \pm 10,5$ & $65,7 \pm 10,0$ & $<0,0001$ \\
\hline $\mathrm{SBP}, \mathrm{mmHg}$ & $107,7 \pm 9,2$ & $107,8 \pm 9,7$ & $107,4 \pm 8,8$ & 0,75 \\
\hline $\mathrm{DBP}, \mathrm{mmHg}$ & $72,0 \pm 7,1$ & $72,4 \pm 6,7$ & $71,4 \pm 7,4$ & 0,38 \\
\hline Urea, $\mathrm{mg} / \mathrm{dL}$ & $24 \pm 4$ & $23 \pm 5$ & $24 \pm 4$ & 0.66 \\
\hline Creatinine, $\mathrm{mg} / \mathrm{dL}$ & $0.7 \pm 0.3$ & $0.7 \pm 0.3$ & $0.6 \pm 0.4$ & 0.36 \\
\hline Progesterone, $\mathrm{ng} / \mathrm{mL}$ & $1 \pm 7$ & $1 \pm 4$ & $1 \pm 11$ & 0.07 \\
\hline
\end{tabular}




\begin{tabular}{lllll}
\hline Variables & Total $(\mathbf{n}=\mathbf{1 8 7})$ & AG $(\mathbf{n}=\mathbf{8 8})$ & SG $(\mathbf{n}=\mathbf{9 9})$ & p-value \\
\hline Exercise (min/session) & ---- & $157 \pm 20.5$ & ---- & -- \\
Exercise (years) & ---- & $5.8 \pm 4.0$ & ---- & --- \\
\hline
\end{tabular}

Values are means_SD. $p<0.05$ Compared by independent t-test. BMI: Body mass index; HR: Heart rate; SBP: Systolic blood pressure; DBP: Diastolic blood pressure; $\mathrm{AG}=$ Active Group; $\mathrm{SG}=$ Sedentary Group.

\subsection{Genotyping Data}

Minor allele frequencies for ADR- $\beta 2$ Gln27Glu polymorphism in the study population were $27 \%$ and $29 \%$, respectively. The genotype distributions were as expected from Hardy-Weinberg equilibrium and were similar between $\mathrm{AG}$ and $\mathrm{SG}$ groups $(\mathrm{p}>0.05)$.

Table 2. Frequencies of ADR- $\beta_{2}$ Gln27Glu polymorphism in physically active and sedentary women.

\begin{tabular}{llllll}
\hline Groups & Genotypes & & & Alleles & \\
\hline & Gln27Gln27 & Gln27Glu27 & Glu27Glu27 & Gln27 & Glu27 \\
\hline GA (99) & $57,57 \%(57)$ & $29,29 \%(29)$ & $13,13 \%(13)$ & $72,2 \%(143)$ & $27,8 \%(55)$ \\
GS ( 88 ) & $59,09 \%(52)$ & $22,72 \%(20)$ & $18,18 \%(16)$ & $70,4 \%(124)$ & $29,6 \%(52)$ \\
& $\chi 2=1.55(2 \mathrm{gl}, \mathrm{p}=0.46)$ & & & $\chi 2=0.14(1 \mathrm{gl}, \mathrm{p}=0.70)$ & \\
\hline
\end{tabular}

Data are presented as No. (\%); $\mathrm{AG}=$ Active Group; $\mathrm{SG}=$ Sedentary Group.

Table 3. Spectral and Nonlinear analysis of heart rate variability according to genotypes ADR- $\beta_{2}$ Gln27Glu polymorphism of the total group in supine position.

\begin{tabular}{llll}
\hline Variables & & Genotypes & p \\
\hline SE & Gln27 Gln27(n=111) & Gln27Glu27(n=49) & Glu27 Glu27(n=29) \\
0V\% & $3.72 \pm 0.38$ & $3.85 \pm 0.31$ & $3.71 \pm 0.34$ \\
1V \% & $16.14 \pm 12.58$ & $12.72 \pm 11.30$ & $17.41 \pm 11.62$ \\
2LV\% & $44.99 \pm 7.05$ & $45.14 \pm 7.02$ & $45.91 \pm 5.86$ \\
2 ULV (\%) & $15.31 \pm 8.57$ & $15.81 \pm 6.66$ & $15.60 \pm 7.19$ \\
NCI & $26.0 \pm 6.20$ & $24.0 \pm 4.55$ & $25.2 \pm 3.53$ \\
CI & $0.72 \pm 0.42$ & $0.75 \pm 0.58$ & 0.11 \\
\hline
\end{tabular}

Values are means \pm SD. $\mathrm{p}<0.05$ compared to ANOVA by Ranks. LF: Low frequency; HF: High frequency; SE: Shannon Entropy; 0V: patterns with no variation; 1V: patterns with one variation; $2 \mathrm{LV}$ : patterns with two like variations; NCI: Normalized Conditional Index; CI: Complexity Index.

The table 3 showed the data from HRV analysis based on symbolic dynamics, Shannon's entropy, complexity index and normalized complexity index of the groups studied. There was no significant difference of any index among genotypes both from the symbolic analysis (SE, $0 \mathrm{~V} \%, 1 \mathrm{~V} \%$, $2 \mathrm{LV} \%$ and $2 \mathrm{ULV} \%)$ and the complexity index ( $>0.05)$.

The relationship between ADR- $\beta_{2}$ Gln27Glu polymorphisms and cardiovascular variables in $\mathrm{AG}$ group and SG are shown in Tables 4. These variants had no effect on symbolic dynamics, SE, complexity index and normalized complexity index. Moreover, the interaction between genotype and exercise did not significantly affect HRV $(\mathrm{p}>0.05)$. AG presented higher values for SE and $2 \mathrm{ULV} \%$ and lower values for $0 \mathrm{~V} \%$ when compared to $\mathrm{SG}(\mathrm{p}<0.05)$. There was no significant difference of a $1 \mathrm{~V} \%, 2 \mathrm{LV} \%$ and NCI index between AG and SG (Table 4).

Table 4. Interactions between exercise and ADR- $\beta_{2}$ Gln27Glu polymorphism on cardiovascular variables in young women.

\begin{tabular}{|c|c|c|c|c|c|c|c|c|}
\hline Groups & Genotypes & ES & $0 \mathrm{~V} \%$ & $1 \mathrm{~V} \%$ & $2 \mathrm{LV} \%$ & $2 \operatorname{ULV}(\%)$ & NCI & CI \\
\hline \multirow[t]{3}{*}{$\mathrm{AG}$} & $\mathrm{G} \ln 27 \mathrm{G} \ln 27(\mathrm{n}=57)$ & $4.0 \pm 0.1$ & $7.3 \pm 4.9$ & $42.0 \pm 6.8$ & $19.8 \pm 7.9$ & $27.0 \pm 2.12$ & $0.77 \pm 0.18$ & $1.22 \pm 0.58$ \\
\hline & Gln27Glu27 $(\mathrm{n}=29)$ & $4.0 \pm 0.1$ & $9.7 \pm 11.9$ & $42.6 \pm 7.5$ & $18.2 \pm 5.9$ & $28.8 \pm 3.10$ & $0.78 \pm 0.25$ & $1.24 \pm 0.62$ \\
\hline & Glu27Glu27 $(n=13)$ & $3.9 \pm 0.07$ & $9.7 \pm 6.1$ & $42.7 \pm 5.1$ & $19.3 \pm 7.0$ & $26.5 \pm 4.35$ & $0.78 \pm 0.41$ & $1.26 \pm 0.46$ \\
\hline \multirow[t]{5}{*}{ SG } & $\mathrm{G} \ln 27 \mathrm{G} \ln 27(\mathrm{n}=52)$ & $3.4 \pm 0.3$ & $21.8 \pm 11.4$ & $47.9 \pm 5.8$ & $11.9 \pm 5.7$ & $16.0 \pm 3.18$ & $0.72 \pm 0.42$ & $1.05 \pm 0.58$ \\
\hline & Gln27Glu27 $(\mathrm{n}=20)$ & $3.2 \pm 0.3$ & $19.0 \pm 8.8$ & $48.7 \pm 4.0$ & $12.3 \pm 6.2$ & $15.8 \pm 5.10$ & $0.73 \pm 0.51$ & $1.07 \pm 0.31$ \\
\hline & $\begin{array}{l}\text { Glu27Glu27 }(\mathrm{n}=16) \\
\mathrm{p} \text { values }\end{array}$ & $3.2 \pm 0.3$ & $23.6 \pm 11.3$ & $48.4 \pm 5.1$ & $12.5 \pm 5.9$ & $15.5 \pm 4.51$ & $0.75 \pm 0.58$ & $1.3 \pm 0.25$ \\
\hline & G & 0.47 & 0.71 & 0.12 & 0.45 & 0.84 & 0.12 & 0.36 \\
\hline & I & 0.34 & 0.95 & 0.41 & 0.96 & 0.70 & 0.52 & 0.56 \\
\hline
\end{tabular}

Values are means $\pm \mathrm{SD}$. AG= Active Group; $\mathrm{SG}=$ Sedentary Group; LF: Low frequency; HF: High frequency; SE: Shannon Entropy; 0V: patterns with no variation; 1V: patterns with one variation; 2LV: patterns with two like variations. $\mathrm{E}=$ exercise; $\mathrm{G}=$ genotype; I=interaction between exercise and genotype. ANOVA two way test $\mathrm{p}<0.05$.

\section{Discussion}

Genetic variations in both peripheral and central $\beta$-AR have major roles in the physiopathology of cardiovascular diseases and in adaptations related to physical exercise because they are also involved in the regulation of cardiac autonomic variation modulations [16,19].

The study sample included active and sedentary women 
in order to verify whether the ADRB2 Glu27Glu polymorphism affects HRV. The sedentary group was classified as having low cardiorespiratory fitness and the physically active group was classified as having regular cardiorespiratory fitness. According to the population studied, our data shows that the level of aerobic physical activity is associated with improvements in parasympathetic modulation and greater complexity. However, the improvements were not enough to show a positive interaction with the polymorphism and were independent of the physical activity status in the population studied.

HRV analysis by means of Shannon's entropy and corrected conditional entropy (complexity index and normalized complexity index) showed no differences in the sequential pattern distribution or in the regularity and predictability of patterns, among the genotypes. Also the symbolic analysis of $0 \mathrm{~V} \%$ and $2 \mathrm{LV} \%$ patterns, regularity and predictability of patterns revealed no differences in HRV ADR $\beta_{2}$ Gln27Gln genotype in both groups.

Atala et al. [16] demonstrated that HRV in time domain was not different between Gln27Glu genotypes subjects. Furthermore, the recessive Glu27Glu and the heterozygous Gln27Glu genotypes had a higher percentage of lowfrequency components (LF\%) than the homozygous Gln27Gln [16]. It was also observed that the Gln27 allele has a protective effect showed by increased parasympathetic modulation in studied individuals; i.e. we can infer that the type of analysis applied influenced the results obtained.

Matsunaga et al. [15] observed that homozygous carriers of the $\beta_{2}$-AR Arg16 allele had lower sympathetic activity than subjects with the Gly allele, and carriers of $\beta_{2}$-AR Glu27 allele were linked with higher cardiac sympathetic modulation than subjects with the Gly allele, and carriers of $\beta_{2}$-AR Glu27 allele were linked with higher cardiac sympathetic autonomic activity. Differences in population or in experimental conditions may have influenced the discrepancy in the results.

The studies reach controversial results due to the many factors under consideration such as the recessive model and homogeneity of the sample (including gender, age and level of trainability of the test subjects) which may easily confound the researchers thus hindering data interpretation.

Recent studies have shown a relationship between aerobic functional capacity and polymorphisms in $\beta_{2}$-AR. Wolfarth et al. [28] observed a positive association between Agr16Gly polymorphism and endurance athletes. Authors have also observed that when comparing carriers and noncarriers of the Gly allele, sedentary individuals present a significant excess amount of said allele, indicating an unfavorable effect during aerobic performance. It is possible that ADR- $\beta_{2}$ influence the variation during aerobic performance due to the contribution in regulating energy use and lipid mobilization in fat tissue. Based on these physiological implications, various studies about the genetic composition of athletes have proposed that alterations in the genetic structure result in an increase of aerobic phenotypes with a higher maximum oxygen use and a rise in fat oxidation. Polymorphism in the Gly16Glu27 haplotype has a relation with positive aerobic phenotypes and helps ideal lipolysis, which can also influence aerobic performance [8]. In contrast, the presence of $\beta$-AR Gln 27 allele was not associated with increased parasympathetic modulation in active women studied.

Meirhaeghe et al. [29] reported that in sedentary men, those carrying the Gln27Gln genotype had higher body weight, BMI and WHR, as compared with Glu27 carriers. Obese individuals with the $A D R \beta_{2}$ Gln27Gln genotype may benefit from physical activity to reduce their weight.

Sedentary behavior has been associated to autonomic alterations with a reduction of parasympathetic activity. Studies among young adults also observed an increase in HF after a 12 week aerobic conditioning among men however, such results were not obtained with women [4]. A study among healthy young women observed a rise in the indexes based on a non-linear analysis (Shannon's Entropy and symbolic patterns) [30]. Additionally, regular physical activity lowers HR during rest because of increased vagal tone [31]. The present study is expanding to include the relationship of this ADR $\beta 2$ Gln27Gln polymorphism to autonomic modulation. The research does not support the hypothesis that active women - those carrying the Gln27Gln genotype - had higher parasympathetic modulation in comparison to Glu27 carriers. These contradictory results may be due to experimental conditions, type of analysis, heterogeneity of genetic variants of the population involved in the studies and the aerobic training status.

Our results confirm other findings reported in the literature, namely, that regular physical exercise is beneficial to the cardiac autonomic nervous regulation. Studies have shown that aerobic physical training promotes high complexity and low regularity of HRV patterns as well as an increase in parasympathetic modulation and a decrease in sympathetic modulation $[4,32,33]$.

However, the level of trainability was not large enough to promote effects of $A D R \beta_{2}$ Gln27Gln polymorphism on parasympathetic modulation in physically active women whereas studies conducted with elite endurance athletes with a high level of trainability had positive results [28]. Studies in large scale are necessary in other populations to elucidate the functional mechanisms of $\beta 2-\mathrm{AR}$ polymorphisms in relation to HR phenotypes and autonomic cardiac modulation.

These results do not support the concept that the genetic polymorphism variation in ADR $\beta 2$ Gln27Gln affects the HRV indexes in physically active or sedentary women. This indicates that other factors might mediate these responses such as the physical training level of women.

\section{Acknowledgements}

This study was supported by CNPq (Conselho Nacional de Desenvolvimento Científico e Tecnológico - Process no. 
370448/2007-3) and FAPESP (Fundação de Amparo à Pesquisa do Estado de São Paulo - Process no. 2006/567881). We are indebted to Dr. Alberto Porta and his team of the Department of Technologies for Health, Galeazzi Orthopaedic Institute, University of Milan, Milan, Italy, for providing the programme for the nonlinear analysis of HRV.

\section{Authors' Contributions}

Rebelo ACS, Melo ABS and Salviati MR were integrated in conception and study design, collection and analysis of data, wrote the first draft of the manuscript and edited and revised subsequent drafts. Verlengia R, Vale AF and Nogueira YL participated in collection of data, and edited and revised the article for important intellectual content. Tamburús NY, Kunz VC and Silva E participated in data collection and edited and revised the article for important. All authors approved the final manuscript as submitted.

\section{References}

[1] Gribble, M. O.; Cheng, A.; Berger, R. D.; Rosman, L.; Guallar, E. Mercury exposure and heart rate variability: A systematic review. Current environmental health reports 2015, 2, 304-314.

[2] Lakusic, N.; Mahovic, D.; Kruzliak, P. Changes in heart rate variability after coronary artery bypass grafting and clinical importance of these findings. 2015, 2015, 680515 .

[3] Montano, N.; Porta, A.; Cogliati, C.; Costantino, G.; Tobaldini, E.; Casali, K. R.; Iellamo, F. Heart rate variability explored in the frequency domain: A tool to investigate the link between heart and behavior. Neuroscience and biobehavioral reviews 2009, 33, 71-80.

[4] Thayer, J. F.; Yamamoto, S. S.; Brosschot, J. F. The relationship of autonomic imbalance, heart rate variability and cardiovascular disease risk factors. International journal of cardiology 2010, 141, 122-131.

[5] Yperzeele, L.; van Hooff, R.J.; Nagels, G.; De Smedt, A.; De Keyser, J.; Brouns, R. Heart rate variability and baroreceptor sensitivity in acute stroke: A systematic review. International journal of stroke : official journal of the International Stroke Society 2015, 10, 796-800.

[6] Su, S.; Lampert, R.; Zhao, J.; Bremner, J. D.; Miller, A.; Snieder, H.; Lee, F.; Khan, D.; Goldberg, J.; Vaccarino, V. Pleiotropy of c-reactive protein gene polymorphisms with creactive protein levels and heart rate variability in healthy male twins. The American journal of cardiology 2009, 104, 1748-1754.

[7] Yang, A. C.; Tsai, S.J.; Hong, C. J.; Wang, C.; Chen, T. J.; Liou, Y. J.; Peng, C. K. Clustering heart rate dynamics is associated with beta-adrenergic receptor polymorphisms: Analysis by information-based similarity index. PloS one 2011, 6, e19232.

[8] Sarpeshkar, V.; Bentley, D. J. Adrenergic-beta(2) receptor polymorphism and athletic performance. Journal of human genetics 2010, 55, 479-485.

[9] Wagoner, L. E.; Craft, L. L.; Singh, B.; Suresh, D. P.; Zengel, P. W.; McGuire, N.; Abraham, W. T.; Chenier, T. C.; Dorn, G.
W., 2nd; Liggett, S. B. Polymorphisms of the beta(2)adrenergic receptor determine exercise capacity in patients with heart failure. Circulation research 2000, 86, 834-840.

[10] Pereira, A.C.; Floriano, M.S.; Mota, G.F.; Cunha, R.S.; Herkenhoff, F.L.; Mill, J.G.; Krieger, J.E. Beta2 adrenoceptor functional gene variants, obesity, and blood pressure level interactions in the general population. Hypertension 2003, 42, 685-692.

[11] Villares, S. M.; Mancini, M. C.; Gomez, S.; Charf, A. M.; Frazzatto, E.; Halpern, A. Associação entre polimorfismo gln27glu do receptor b2-adrenérgico e hipertensão arterial sistêmica em obesos mórbidos. Arquivos Brasileiros de Endocrinologia \& Metabologia 2000, 44, 72-80.

[12] Dorn, G. W., 2nd. Adrenergic signaling polymorphisms and their impact on cardiovascular disease. Physiological reviews 2010, 90, 1013-1062.

[13] Nakashima, H.; Omae, K.; Nomiyama, T.; Yamano, Y.; Takebayashi, T.; Sakurai, Y. Beta-3-adrenergic receptor trp64arg polymorphism: Does it modulate the relationship between exercise and percentage of body fat in young adult japanese males? Environmental health and preventive medicine 2013, 18, 323-329.

[14] Masuo, K.; Lambert, G. W. Relationships of adrenoceptor polymorphisms with obesity. Journal of obesity 2011, 2011, 609485 .

[15] Matsunaga, T.; Yasuda, K.; Adachi, T.; Gu, N.; Yamamura, T.; Moritani, T.; Tsujimoto, G.; Tsuda, K. Association of betaadrenoceptor polymorphisms with cardiac autonomic modulation in japanese males. American heart journal 2007, $154,759-766$.

[16] Atala, M. M.; Goulart, A.; Guerra, G. M.; Mostarda, C.; Rodrigues, B.; Mello, P. R.; Casarine, D.E.; Irigoyen, M. C.; Pereira, A. C.; Consolim-Colombo, F.M. Arg16gly and gln27glu beta2 adrenergic polymorphisms influence cardiac autonomic modulation and baroreflex sensitivity in healthy young brazilians. American journal of translational research 2015, 7, 153-161.

[17] Bouchard, C. Genomic predictors of trainability. Experimental physiology 2012, 97, 347-352.

[18] Macho-Azcarate, T.; Marti, A.; Calabuig, J.; Martinez, J.A. Basal fat oxidation and after a peak oxygen consumption test in obese women with a beta2 adrenoceptor gene polymorphism. The Journal of nutritional biochemistry 2003, 14, 275-279.

[19] Castellano, M.; Rossi, F.; Giacche, M.; Perani, C.; Rivadossi, F.; Muiesan, M.L.; Salvetti, M.; Beschi, M.; Rizzoni, D.; Agabiti-Rosei, E. Beta(2)-adrenergic receptor gene polymorphism, age, and cardiovascular phenotypes. Hypertension 2003, 41, 361-367.

[20] Porta, A.; Tobaldini, E.; Guzzetti, S.; Furlan, R.; Montano, N.; Gnecchi-Ruscone, T. Assessment of cardiac autonomic modulation during graded head-up tilt by symbolic analysis of heart rate variability. American journal of physiology. Heart and circulatory physiology 2007, 293, H702-708.

[21] Heart rate variability. Standards of measurement, physiological interpretation, and clinical use. Task force of the european society of cardiology and the north american society of pacing and electrophysiology. European heart journal 1996, 17, 354-381. 
[22] Porta, A.; Guzzetti, S.; Montano, N.; Furlan, R.; Pagani, M.; Malliani, A.; Cerutti, S. Entropy, entropy rate, and pattern classification as tools to typify complexity in short heart period variability series. IEEE transactions on bio-medical engineering 2001, 48, 1282-1291.

[23] Porta, A.; Faes, L.; Mase, M.; D'Addio, G.; Pinna, G. D.; Maestri, R.; Montano, N.; Furlan, R.; Guzzetti, S.; Nollo, G. et al. An integrated approach based on uniform quantization for the evaluation of complexity of short-term heart period variability: Application to $24 \mathrm{~h}$ holter recordings in healthy and heart failure humans. Chaos (Woodbury, N.Y.) 2007, 17, 015117

[24] Porta, A.; Baselli, G.; Liberati, D.; Montano, N.; Cogliati, C.; Gnecchi-Ruscone, T.; Malliani, A.; Cerutti, S. Measuring regularity by means of a corrected conditional entropy in sympathetic outflow. Biological cybernetics 1998, 78, 71-78.

[25] Salazar, L.A.; Hirata, M.H.; Cavalli, S.A.; Machado, M.O.; Hirata, R.D. Optimized procedure for DNA isolation from fresh and cryopreserved clotted human blood useful in clinical molecular testing. Clinical chemistry 1998, 44, 1748-1750.

[26] Rebelo, A.C.; Verlengia, R.; Kunz, V.; Tamburus, N.; Cerda, A.; Hirata, R.; Hirata, M.; Silva, E. Lack association of estrogen receptor alpha gene polymorphisms with cardiorespiratory and metabolic variables in young women. International Journal of Molecular Sciences 2012, 13, 1369113703.

[27] Dias, R.G.; Pereira, A.d.C.; Negrão, C.E.; Krieger, J.E. Polimorfismos genéticos determinantes da performance física em atletas de elite. Revista Brasileira de Medicina do Esporte 2007, 13, 209-216.
[28] Wolfarth, B.; Rankinen, T.; Muhlbauer, S.; Scherr, J.; Boulay, M.R.; Perusse, L.; Rauramaa, R.; Bouchard, C. Association between a beta2-adrenergic receptor polymorphism and elite endurance performance. Metabolism: clinical and experimental 2007, 56, 1649-1651.

[29] Meirhaeghe, A.; Helbecque, N.; Cottel, D.; Amouyel, P. Beta2-adrenoceptor gene polymorphism, body weight, and physical activity. Lancet 1999, 353, 896.

[30] Rebelo, A.C.; Tamburus, N.; Salviati, M.; Celante, V.; Takahashi, A.; de Sa, M.F.; Catai, A.; Silva, E. Influence of third-generation oral contraceptives on the complexity analysis and symbolic dynamics of heart rate variability. The European journal of contraception \& reproductive health care : the official journal of the European Society of Contraception 2011, 16, 289-297.

[31] Marães, V.R.F.S. Frequência cardíaca e sua variabilidade: Análises e aplicações. Revista Andaluza de Medicina del Deporte 2010, 03, 33-42.

[32] Clemente-Suarez, V.J.; Fernandes, R.J.; Arroyo-Toledo, J.J.; Figueiredo, P.; Gonzalez-Rave, J.M.; Vilas-Boas, J.P. Autonomic adaptation after traditional and reverse swimming training periodizations. Acta physiologica Hungarica 2015, 102, 105-113.

[33] Rezende Barbosa, M.P.; Netto Junior, J.; Cassemiro, B.M.; de Souza, N.M.; Bernardo, A.F.; da Silva, A.K.; Pastre, C.M.; Vanderlei, L.C. Impact of functional training on cardiac autonomic modulation, cardiopulmonary parameters and quality of life in healthy women. Clinical physiology and functional imaging 2015. 